\title{
On the catalysis of the electroweak vacuum decay by black holes at high temperature
}

\author{
D. Canko ${ }^{1}$, I. Gialamas ${ }^{1}$, G. Jelic-Cizmek ${ }^{2}$, A. Riotto ${ }^{2}$, N. Tetradis ${ }^{1, a}$ \\ ${ }^{1}$ Department of Physics, National and Kapodistrian University of Athens, Zographou, 15784 Athens, Greece \\ ${ }^{2}$ Department of Theoretical Physics and Center for Astroparticle Physics (CAP), University of Geneva, 24 quai E. Ansermet, 1211 Geneva 4 , \\ Switzerland
}

Received: 4 February 2018 / Accepted: 16 April 2018 / Published online: 23 April 2018

(C) The Author(s) 2018

\begin{abstract}
We study the effect of primordial black holes on the classical rate of nucleation of AdS regions within the standard electroweak vacuum at high temperature. We base our analysis on the assumption that, at temperatures much higher than the Hawking temperature, the main effect of the black hole is to distort the Higgs configuration dominating the transition to the new vacuum. We estimate the barrier for the transition by the ADM mass of this configuration, computed through the temperature-corrected Higgs potential. We find that the exponential suppression of the nucleation rate can be reduced significantly, or even eliminated completely, in the black-hole background if the Standard Model Higgs is coupled to gravity through the renormalizable term $\xi \mathcal{R} h^{2}$.
\end{abstract}

\section{Introduction}

It is well-known that for the current measured values of the Higgs and top quark masses the Standard Model (SM) effective potential develops an instability. Due to the running of the quartic coupling, the effective potential of the Higgs reaches a maximum and then becomes unbounded from below at values of the Higgs field of about $5 \times 10^{10} \mathrm{GeV}$. Our electroweak vacuum has a lifetime which is many orders of magnitude larger than the age of the Universe and is therefore stable against decay through quantum tunnelling.

A pertinent question concerns the fate of the electroweak vacuum during the evolution of the Universe in situations in which gravity plays a pronounced role. The gravitational background can have a significant effect on the rate of vacuum decay, leading to its enhancement or suppression [1]. This issue is crucial for the decay of the electroweak vacuum, because of the extreme sensitivity of the Higgs potential to the Higgs and top masses [2-17]. The fact that the

\footnotetext{
a e-mail: ntetrad@phys.uoa.gr
}

gravitational effects are significant during inflation has led to an extensive investigation of the stability of the electroweak vacuum during this era, leading to bounds on the inflationary scale $[14,18-35]$ in order to avoid a catastrophic singularity characterized as the AdS crunch $[1,31,36]$.

Strong gravitational fields are also sourced by black holes, which raises the question of electroweak vacuum stability in their vicinity. It has been argued that black holes can act as impurities enhancing the quantum decay rate to a level incompatible with the age of the Universe [37-39]. It is natural to expect a similar enhancement for classical transitions to the true Higgs vacuum, induced by the high-temperature environment of the early Universe [40]. This second mechanism can be explored through a more intuitive approach, with fewer technical uncertainties than the calculation of the quantum tunnelling rate. In any case, the question whether the existence of primordial black holes [41-44] is consistent with the stability of the electroweak vacuum has important implications for the compatibility of the Standard Model of particle physics and the cosmological model [45].

In this paper we explore further the scenario of temperatureinduced vacuum decay in the black-hole background [40]. Firstly, we repeat the analysis using the temperature-corrected effective potential of the Higgs field, instead of the zerotemperature one employed in [40]. Secondly, we allow for a nonminimal coupling of the Higgs field to gravity. This additional coupling can have strong effects on the action of the bounce in the case of quantum tunnelling, or the free energy of the critical bubble in the case of thermal tunnelling. Such behavior is known to occur in the context of inflation [31] and similar features are expected within the strong gravitational field of a black hole.

The paper is organized as follows. In Sect. 2 we derive the equations of motion and the relevant expressions for the energy of the bubble configuration. In Sect. 3 we study the finite-temperature effects on the Higgs potential and estimate 
the size of the barrier to be overcome for vacuum decay in the presence of black holes. In Sect. 4 we discuss the stability of the electroweak vacuum and derive constraints for the allowed range of the nonminimal coupling of the Higgs field to gravity. In the final section we give our conclusions.

\section{Equations of motion}

Our starting point is the action of the SM Higgs coupled to gravity, where we also allow the renormalizable term whose strength is parametrized by the coupling $\xi$

$S=\int_{\mathcal{M}} d^{4} x \sqrt{-g}\left(-\frac{1}{2}(\nabla h)^{2}-V(h)+\frac{1}{16 \pi G} \mathcal{R}+\frac{1}{2} \xi h^{2} \mathcal{R}\right)$.

The equations of motion are

$$
\begin{aligned}
& \left(\frac{1}{8 \pi G}+\xi h^{2}\right)\left(\mathcal{R}_{\mu \nu}-\frac{1}{2} g_{\mu \nu} \mathcal{R}\right) \\
& =\nabla_{\mu} h \nabla_{\nu} h-g_{\mu \nu}\left(\frac{1}{2}(\nabla h)^{2}+V(h)\right) \\
& \quad+2 \xi\left(\nabla_{\mu}\left(h \nabla_{\nu} h\right)-g_{\mu \nu} \nabla_{\lambda}\left(h \nabla^{\lambda} h\right)\right), \\
& \nabla_{\mu} \nabla^{\mu} h+\xi h \mathcal{R}=\frac{d V(h)}{d h} .
\end{aligned}
$$

The use of the equations of motion allows to simplify the action further. In particular, taking the trace of Eq. (2) expresses the curvature scalar $R$ in terms the scalar field. The derivative of the potential can be eliminated through Eq. (3). One eventually finds

$$
\begin{aligned}
\mathcal{R}\left(\frac{1}{8 \pi G}+\xi h^{2}\right)= & (1+6 \xi)(\nabla h)^{2}+4 V(h) \\
& +6 \xi h \nabla_{\mu} \nabla^{\mu} h .
\end{aligned}
$$

Substitution in Eq. (1) gives

$S=\int_{\mathcal{M}} d^{4} x \sqrt{-g}\left(V(h)+3 \xi \nabla_{\mu}\left(h \nabla^{\mu} h\right)\right)$.

The second term in the above expression is reduced to a boundary term upon integration.

The presence of the horizon at $r=R_{\mathrm{h}}$ imposes specific boundary conditions for the Higgs field at this point in order that it stays finite. Moreover, the consistency of the variational principle in the context of gravity requires the presence of a boundary term [46]

$S_{b}=2 \epsilon \int_{\partial \mathcal{M}} d^{3} y \sqrt{-g_{\text {ind }}}\left(\frac{1}{16 \pi G}+\frac{1}{2} \xi h^{2}\right) \mathcal{K}$,

with $\left(g_{\text {ind }}\right)_{\alpha \beta}$ the induced metric on the boundary surface, $\epsilon= \pm 1$ depending on whether the surface is timelike or spacelike, respectively, and $\mathcal{K}$ the trace of the extrinsic curvature. We solve the equations of motion, starting slightly outside the horizon, assuming the presence of such a term with $\epsilon=-1$. The boundary term makes the action $\xi$-dependent even for solutions with $\mathcal{R}=0$, for which the bulk contribution (1) does not depend on $\xi$. This feature can be reflected in the ADM mass, as we shall see in the following.

We consider now the SM Higgs field in the presence of black holes that can trigger the decay of the electroweak vacuum by providing nucleation sites [37-39]. The decay takes place through the formation of bubbles of the new vacuum around the black holes. The critical bubble is a static configuration, whose profile can be obtained by solving the equation of motion of the Higgs field with appropriate boundary conditions. The field interpolates between values on either side of the maximum of the potential.

In a thermal environment, we expect that the probability for the transition to the true vacuum will be exponentially suppressed, with the exponent being proportional to $1 / T$. When the effects of gravity and entropy are negligible, the proportionality factor is simply the energy of the dominant saddle point underlying the transition. If the entropy is large, the energy is replaced by the free energy. In the presence of significant gravitational effects, however, the generalization is not obvious. We shall assume here that the ADM mass of the critical Higgs bubble is a measure of the barrier that must be overcome for the field to fluctuate beyond the potential maximum. In order to take into account the entropy associated with the heat bath, we shall employ the temperaturecorrected Higgs potential, which accounts for the entropy density of the thermally excited Higgs particles.

The appropriate metric for our analysis has the form

$$
\begin{aligned}
\mathrm{d} s^{2}= & -N(r) e^{2 \delta(r)} \mathrm{d} t^{2}+N^{-1}(r) \mathrm{d} r^{2} \\
& +r^{2}\left(\mathrm{~d} \theta^{2}+\sin ^{2} \theta \mathrm{d} \phi^{2}\right), \\
N(r)= & 1-2 \frac{G M(r)}{r} .
\end{aligned}
$$

With this ansatz, one has to solve the Einstein and Higgsfield equations for the static critical bubbles. The function $N(r)$ vanishes on the horizon, which gives $2 G M\left(R_{\mathrm{h}}\right)=R_{\mathrm{h}}$. The quantity $R_{\mathrm{h}} /(2 G)$ can be identified with the black-hole mass $M_{\text {bh }}$. In order to obtain a finite ADM mass for the bubble configuration we must avoid a singularity in Eq. (9) at $r=R_{\mathrm{h}}$. This can be achieved by choosing $h^{\prime}\left(R_{\mathrm{h}}\right)$ appropriately. We also require that $h(r) \rightarrow 0$ for $r \rightarrow \infty$. We are interested in the quantity $\delta M(r)=M(r)-R_{\mathrm{h}} /(2 G)$. The asymptotic value $\delta M_{\text {tot }} \equiv \delta M(\infty)$ gives an estimate of the barrier associated with the production of the bubble around a central black hole with horizon radius $R_{\mathrm{h}}$. The characteristic scale of the solutions is set by the largest of $h_{\max }$, the value of the SM Higgs at the maximum of its potential ${ }^{1}$ and the temperature $T$, which are expected to be much smaller than the

\footnotetext{
${ }^{1}$ While this definition is not gauge-invariant, one could define $h_{\max }$ as $V_{\max }^{1 / 4}$ which, thanks to the Nielsen identity, is gauge-invariant [47].
} 
Planck scale $M_{\mathrm{Pl}}$. This leads to a significant simplification of the setup, resulting from the fact that $\delta M(r) \ll R_{\mathrm{h}} /(2 G)$.

Since the black-hole mass is the leading factor determining the gravitational background, we write $M(r)=$ $R_{\mathrm{h}} /(2 G)+\delta M(r)$ and keep the leading contributions in $G$. We find that the equations of motion are reduced to

$$
\begin{aligned}
h^{\prime \prime}+( & \left.\frac{2}{r}+\frac{R_{\mathrm{h}}}{r\left(r-R_{\mathrm{h}}\right)}\right) h^{\prime}=\frac{r}{r-R_{\mathrm{h}}} \frac{d V(h)}{d h}, \\
\delta M^{\prime}= & 4 \pi r^{2}\left(\frac{1}{2} \frac{r-R_{\mathrm{h}}}{r} h^{\prime 2}+V(h)\right) \\
& +4 \xi \pi r^{2}\left(2 \frac{r-R_{\mathrm{h}}}{r} h^{\prime 2}+2 h \frac{d V(h)}{d h}-\frac{R_{\mathrm{h}}}{r^{2}} h h^{\prime}\right),
\end{aligned}
$$

with the prime denoting a derivative with respect to $r$. The equation for $\delta$ is reduced to $\delta^{\prime}=0$, and we set $\delta=1$. Avoiding the singularity at $r=R_{\mathrm{h}}$ in Eq. (8) requires that we impose

$h^{\prime}\left(R_{\mathrm{h}}\right)=R_{\mathrm{h}} \frac{d V\left(h\left(R_{\mathrm{h}}\right)\right)}{d h}$.

This boundary condition correctly reproduces the standard condition $h^{\prime}(0)=0$ in the absence of a black hole. Our definition of $\delta M(r)$ imposes $\delta M\left(R_{\mathrm{h}}\right)=0$. The value of $h\left(R_{\mathrm{h}}\right)$ must be tuned so that the condition $h(r) \rightarrow 0$ for $r \rightarrow \infty$ is satisfied.

The form of the above equations demonstrates that the Higgs configuration is not modified by the nonminimal coupling to gravity, and, therefore, is independent of $\xi$. The bubble has a negligible effect on the gravitational background, which is determined by the black-hole mass to a very good approximation. Allowing for a nonminimal Higgs coupling to gravity through a term $\sim h^{2} \mathcal{R}$ in the action does not modify this conclusion, because the curvature $\mathcal{R}$ vanishes in a black-hole background. As a result, all modifications to the background due to the Higgs are suppressed by $M_{\mathrm{Pl}}$.

However, the mass of the bubble configuration receives a correction proportional to $\xi$. The mass can be expressed as

$\delta M_{\mathrm{tot}}=F_{1}\left(R_{\mathrm{h}}\right)+\xi F_{2}\left(R_{\mathrm{h}}\right)$,

with

$$
\begin{aligned}
& F_{1}\left(R_{\mathrm{h}}\right)=4 \pi \int_{R_{\mathrm{h}}}^{\infty} d r r^{2}\left(\frac{1}{2} \frac{r-R_{\mathrm{h}}}{r} h^{\prime 2}+V(h)\right) \\
& F_{2}\left(R_{\mathrm{h}}\right)=4 \pi \int_{R_{\mathrm{h}}}^{\infty} d r r^{2}\left(2 \frac{r-R_{\mathrm{h}}}{r} h^{\prime 2}+2 h \frac{d V(h)}{d h}-\frac{R_{\mathrm{h}}}{r^{2}} h h^{\prime}\right) .
\end{aligned}
$$

It is possible to put the above expressions in an alternative form. The equation of motion (8) can be written as

$$
\left(r\left(r-R_{\mathrm{h}}\right) h^{\prime}\right)^{\prime}=r^{2} \frac{d V(h)}{d h} .
$$

Substitution into the expression (12) gives

$$
\begin{aligned}
F_{1}\left(R_{\mathrm{h}}\right)= & 4 \pi \int_{R_{\mathrm{h}}}^{\infty} d r\left[\frac{1}{2}\left(r\left(r-R_{\mathrm{h}}\right) h h^{\prime}\right)^{\prime}\right. \\
& \left.-\frac{1}{2} r^{2} h \frac{d V(h)}{d h}+r^{2} V(h)\right] \\
= & 4 \pi \int_{R_{\mathrm{h}}}^{\infty} d r r^{2}\left(-\frac{1}{2} h \frac{d V(h)}{d h}+V(h)\right) .
\end{aligned}
$$

Also, the use of Eqs. (14) in (13) gives

$$
\begin{aligned}
F_{2}\left(R_{\mathrm{h}}\right) & =4 \pi \int_{R_{\mathrm{h}}}^{\infty} d r\left(2 r\left(r-R_{\mathrm{h}}\right) h h^{\prime}-\frac{1}{2} R_{\mathrm{h}} h^{2}\right)^{\prime} \\
& =2 \pi R_{\mathrm{h}} h^{2}\left(R_{\mathrm{h}}\right) .
\end{aligned}
$$

The mass receives a contribution $\sim \xi h^{2}\left(R_{\mathrm{h}}\right) R_{\mathrm{h}}$, which reflects the presence of the boundary term (6) in the action.

In Ref. [40] the zero-temperature potential was used in order to compute the mass of the bubble configuration as a function of the black-hole mass for $\xi=0$. The bubble mass was then used in order to estimate the vacuum decay rate at non-zero temperature. In the following section we shall improve on this estimate by considering the temperaturecorrected Higgs potential, which corresponds to the freeenergy density of the thermal bath. Before doing so, we present here the generalization of the results of Ref. [40] for nonzero $\xi$, still using the zero-temperature Higgs potential. We employ the approximate form $V \sim \lambda(h) h^{4} / 4$, which is a good approximation for values of the Higgs field around its maximum at $h_{\max } \sim 5 \times 10^{10} \mathrm{GeV}$. The quartic coupling $\lambda$ varies from 0.02 to -0.02 for Higgs values between $10^{6} \mathrm{GeV}$ and $10^{20} \mathrm{GeV}$, respectively. Near the maximum the potential can be approximated as [31]

$V(h) \simeq-b \ln \left(\frac{h^{2}}{h_{\max }^{2} \sqrt{e}}\right) \frac{h^{4}}{4}, \quad b \simeq 0.16 /(4 \pi)^{2}$.

In Fig. 1 we plot the functions $F_{1}$ and $F_{2}$ that determine the bubble mass $\delta M_{\text {tot }}$, as a function of the black-hole radius. All quantities are measured in units of $h_{\max }$. We have normalized $F_{1}\left(R_{\mathrm{h}}\right)$ with respect to its value $F_{1}(0)$ in the absence of a black hole and we depict it in the left panel of Fig. 1. It is apparent that for $\xi=0$ the ADM mass of the bubble configuration is reduced by an approximate factor of 2 within a certain range of black-hole masses around $R_{\mathrm{h}}=10 h_{\max }$. In the right panel of Fig. 1 we depict the ratio $F_{2}\left(R_{\mathrm{h}}\right) / F_{1}\left(R_{\mathrm{h}}\right)$. It vanishes for $R_{\mathrm{h}} \rightarrow 0$, but quickly grows and remains almost constant for $R_{\mathrm{h}} \gtrsim 20 h_{\max }$, with a value $\simeq 6.6$.

\section{Finite-temperature effects}

The next step is to consider the immersion of the black holeHiggs system in the thermal environment of the early Universe. In a thermal environment, the quantity relevant for 

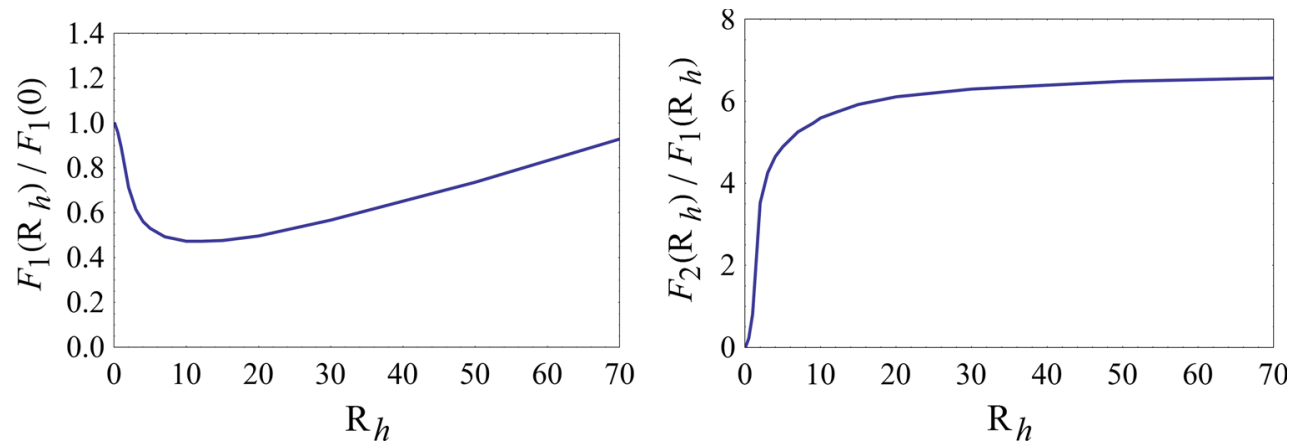

Fig. 1 The quantities $F_{1}$ and $F_{2}$, that determine the bubble mass $\delta M_{\text {tot }}$, as a function of the black-hole radius. All quantities in units of the value of the Higgs field $h_{\max }$ at the maximum of the potential

transitions between different states is the free energy, which accounts for the effect of entropy. In this respect, it is natural to employ the temperature-dependent effective potential, which can be identified with the free-energy density. On the other hand, the gravitational field is sourced by the energymomentum tensor, which includes the energy density and is identified with the zero-temperature potential. The formal derivation of the appropriate expressions on a strong gravitational background is difficult. For example, the effects of temperature are usually taken into account by compactifying the Euclidean time direction. On a black-hole background, the compactification scale is set by the Hawking temperature, which does not coincide necessarily with the ambient temperature, leading to singularities in the underlying geometry. For these reasons our approach will be intuitive rather than formal.

As we saw in the previous section, the backreaction of the Higgs field is negligible, so that the background is described by the Schwarzschild metric to a very good approximation. The equations relevant for our problem, Eqs. (8) and (9), determine the shape and energy of the Higgs configuration that the fluctuating system on this background has to go through for the transition to occur. At nonzero temperature, energy is expected to be replaced by free energy as the relevant quantity in the calculation of the nucleation rate. It is then justifiable intuitively to replace the zero-temperature potential with the high-temperature one. The resulting equations have the correct limits for vanishing temperature or blackhole mass. We emphasize, however, that a formal derivation is lacking.

The characteristic scale of the solutions is set by the largest of $h_{\max }$ and the temperature $T$. For $T \gg h_{\max }$, the form of the zero-temperature potential near its maximum at $h_{\max }$ is not relevant for our calculation. The temperature effects shift the maximum of the potential to a value proportional to $T$. The only dimensionful scale in the problem is the temperature, which determines the scale at which the running couplings must be evaluated. The temperature corrections to the Higgs potential are summarized in Ref. [16]. The Higgs field devel- ops a thermal mass $m_{\mathrm{T}}^{2}=\kappa^{2} T^{2}$, with

$$
\begin{aligned}
\kappa^{2}= & \frac{1}{12}\left(\frac{3}{4} g^{\prime 2}+\frac{9}{4} g^{2}+3 y_{\mathrm{t}}^{2}+6 \lambda\right) \\
& -\frac{1}{32 \pi} \sqrt{\frac{11}{6}\left(g^{\prime 3}+3 g^{3}\right)} \\
& -\frac{3}{16 \pi} \lambda \sqrt{g^{\prime 2}+3 g^{2}+8 \lambda+4 y_{t}^{2}} .
\end{aligned}
$$

The running gauge, $\tau$-Yukawa and quartic running couplings must be evaluated at a scale set by the temperature.

A semi-analytical treatment of the effects on thermal tunnelling arising from the black-hole background and the nonminimal Higgs-gravity coupling is possible if we approximate the full potential as

$V(h, T) \simeq \frac{1}{2} \kappa^{2} T^{2} h^{2}+\frac{1}{4} \lambda h^{4}$.

The quartic $\lambda$ is taken to be constant with a value corresponding to the running coupling at a scale set by the temperature. In the temperature range between $10^{14}$ and $10^{18} \mathrm{GeV}$, we have $\kappa \simeq 0.3$, with a $10 \%$ decrease for increasing $T$. Also, $\lambda \simeq-0.015$ with a $15 \%$ decrease for increasing $T$ [16]. Neglecting the logarithmic running of the quartic coupling is a reasonable approximation. Our discussion can be repeated easily for lower reheating temperatures, but a fully numerical analysis is necessary if quantitative precision is required.

Through the rescalings

$h=\frac{\kappa T}{\sqrt{|\lambda|}} \tilde{h}, \quad r=\frac{1}{\kappa T} \tilde{r}$,

the total free energy of the bubble configuration can be expressed as $\kappa T /|\lambda|$ times a numerical factor [48]. Implementing the above, along with

$R_{\mathrm{h}}=\frac{1}{\kappa T} \tilde{R}_{\mathrm{h}} \quad V(h, T)=\frac{\kappa^{4} T^{4}}{|\lambda|} \tilde{V}(\tilde{h})$,

results in Eq. (8) being replaced by a similar equation for the rescaled variables and $\tilde{V}(\tilde{h})=\tilde{h}^{2} / 2-\tilde{h}^{4} / 4$. Its solution is presented in Fig. 2 for three different black-hole masses. It is 


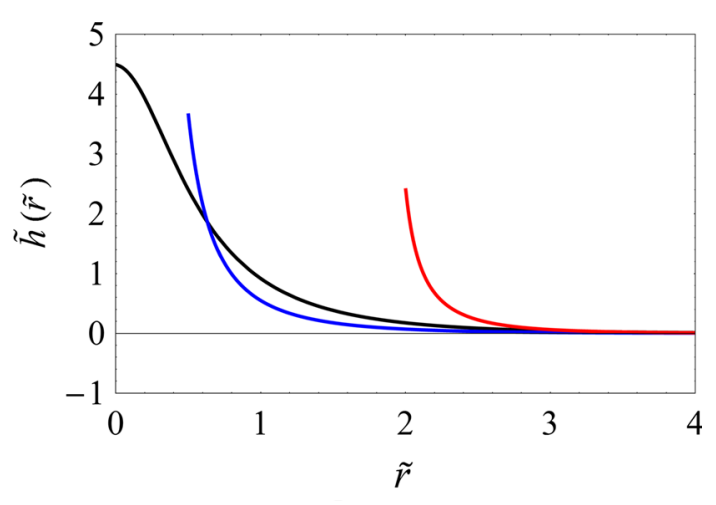

Fig. 2 The rescaled Higgs field outside a black hole with rescaled radius $\tilde{R}_{h}=0.01,0.5,2$ (lines from left to right)

apparent that the characteristic scale of the Higgs configuration is comparable to the horizon radius for $\tilde{R}_{h}=\mathcal{O}(1)$. For such black holes, the Hawking temperature satisfies $T_{\mathrm{H}} / T=\kappa /\left(4 \pi \tilde{R}_{\mathrm{h}}\right)=\mathcal{O}\left(10^{-2}\right)$. There is no thermal equilibrium between the black hole and the environment, while the effect of the Hawking radiation on the ambient thermal bath can be neglected. We shall see below that black holes with $\tilde{R}_{h} \simeq 0.5$ have a strong effect on the vacuum decay rate [40]. This gravitational effect is localized in the vicinity of the black hole. As noted in Ref. [45], the analysis of the quantum decay rate [37-39] results in an expression that indicates that the compactification of the Euclidean time direction at a radius $\sim 1 / T_{\mathrm{H}}$, linked to the Hawking temperature, plays a role at arbitrarily large distances from the black hole. The effect we are discussing is not of similar nature, and has a more robust intuitive interpretation.

The free energy of the bubble configuration can be put in the form

$\frac{\delta M_{\mathrm{tot}}}{T}=\frac{\kappa}{|\lambda|}\left(F_{1}\left(\tilde{R}_{\mathrm{h}}\right)+\xi F_{2}\left(\tilde{R}_{\mathrm{h}}\right)\right)$,

with $\kappa /|\lambda| \sim 20$ and

$$
\begin{aligned}
& F_{1}\left(\tilde{R}_{\mathrm{h}}\right)=4 \pi \int_{\tilde{R}_{\mathrm{h}}}^{\infty} d \tilde{r} \tilde{r}^{2}\left(-\frac{1}{2} \tilde{h} \frac{d \tilde{V}(\tilde{h})}{d \tilde{h}}+\tilde{V}(\tilde{h})\right) \\
& =4 \pi \int_{\tilde{R}_{\mathrm{h}}}^{\infty} d \tilde{r} \tilde{r}^{2} \frac{1}{4} \tilde{h}^{4} . \\
& F_{2}\left(\tilde{R}_{\mathrm{h}}\right)=2 \pi \tilde{R}_{\mathrm{h}} \tilde{h}^{2}\left(\tilde{R}_{\mathrm{h}}\right) .
\end{aligned}
$$

We find $F_{1}(0) \simeq 18.9$, in agreement with Ref. [48]. Also $F_{2}(0)=0$, as can be checked through a partial integration, use of the equation of motion, and remembering that $h^{\prime}(0)=0$ for $R_{\mathrm{h}}=0$. Our approximations result in a value for the ratio $\delta M_{\text {tot }} / T=18.9 \kappa /|\lambda|$ in the absence of a black hole that is independent of the temperature: $\delta M_{\mathrm{tot}} / T \simeq 380$. The complete analysis of the renormalized action of the SM Higgs field is consistent with this value, but also indicates a $20 \%$ decrease of the action for temperatures between $10^{14}$ and $10^{18} \mathrm{GeV}[16,17]$. At lower temperatures, the logarithmic running of the quartic term in the potential becomes important and numerical determination of the bubble profile is needed for every value of $T$. However, we expect that the effect of the black hole on the ratio $\delta M_{\text {tot }} / T$ can be described again by a multiplicative factor $\left(F_{1}\left(\tilde{R}_{\mathrm{h}}\right)+\xi F_{2}\left(\tilde{R}_{\mathrm{h}}\right)\right) / F_{1}(0)$, as in Eq. (22). This expectation is supported by the analysis of Ref. [40], in which the zero-temperature potential was used.

In Fig. 3 we depict the functions $F_{1}\left(\tilde{R}_{\mathrm{h}}\right)$ and $F_{2}\left(\tilde{R}_{\mathrm{h}}\right)$. We have normalized $F_{1}\left(\tilde{R}_{\mathrm{h}}\right)$ with respect to its value $F_{1}(0)$ in the absence of a black hole. The form of $F_{1}\left(\tilde{R}_{\mathrm{h}}\right)$ is similar to that observed in Ref. [40]: the free energy of the bubble configuration for $\xi=0$ is reduced by an approximate factor of 2 within a certain range of black-hole masses around $R_{\mathrm{h}}=$ $0.5 /(\kappa T) \simeq 1.7 / T$. As a result, the nucleation rate for $\xi=0$ can be reduced significantly in the black-hole background. In the right panel of Fig. 3 we depict the ratio $F_{2}\left(\tilde{R}_{\mathrm{h}}\right) / F_{1}\left(\tilde{R}_{\mathrm{h}}\right)$. It vanishes for $\tilde{R}_{\mathrm{h}} \rightarrow 0$, but quickly grows and remains almost constant for $\tilde{R}_{\mathrm{h}} \gtrsim 0.5$, with a value $\simeq 5.3$.

A very interesting feature is the effect of the nonminimal coupling on the bubble free energy. The dependence on $\xi$ is linear, as can be seen from Eq. (22). Large positive values of $\xi$ result in the growth of $\delta M_{\mathrm{tot}} / T$ and the suppression of the nucleation rate. From Eq. (22) it is apparent that, for every black-hole mass at a given temperature, there is a critical negative value $\xi_{\text {cr }}=-F_{1}\left(\tilde{R}_{\mathrm{h}}\right) / F_{2}\left(\tilde{R}_{\mathrm{h}}\right)$, for which the exponential suppression of the nucleation rate is eliminated. Clearly, the saddle-point approximation breaks down before this point is reached. On the other hand, the probability of vacuum decay in the early Universe depends also on the total number of primordial black holes that can be generated. In the following section we discuss this point in detail.

\section{Vacuum (in)stability in the presence of black holes}

The nucleation probability per unit time in the background of a black hole during the radiation-dominated era of the early Universe is $d P / d t \sim T \exp \left(-\delta M_{\text {tot }} / T\right)$. There is no volume factor because of the absence of translational invariance. The characteristic time interval that can be associated with the temperature scale $T$ is the Hubble time $1 / H \sim M_{\mathrm{Pl}} / T^{2}$. The nucleation can be efficient over longer times, but we are interested in a lower bound for the probability. Neglecting the evaporation of the black hole, we have a bubble-nucleation probability $P \sim M_{\mathrm{Pl}} / T \exp \left(-\delta M_{\mathrm{tot}} / T\right)$. The most uncertain factor in our discussion is the number of primordial black holes within our past light cone. The number $N$ of causally independent regions at a time during the radiation-dominated era with temperature $T$, which are currently within our horizon, is $N \sim 10^{34}(T / \mathrm{GeV})^{3}$ [40]. One needs to estimate the probability $p$ for a black hole to exist within one of these regions. This is a strongly model-dependent quantity 

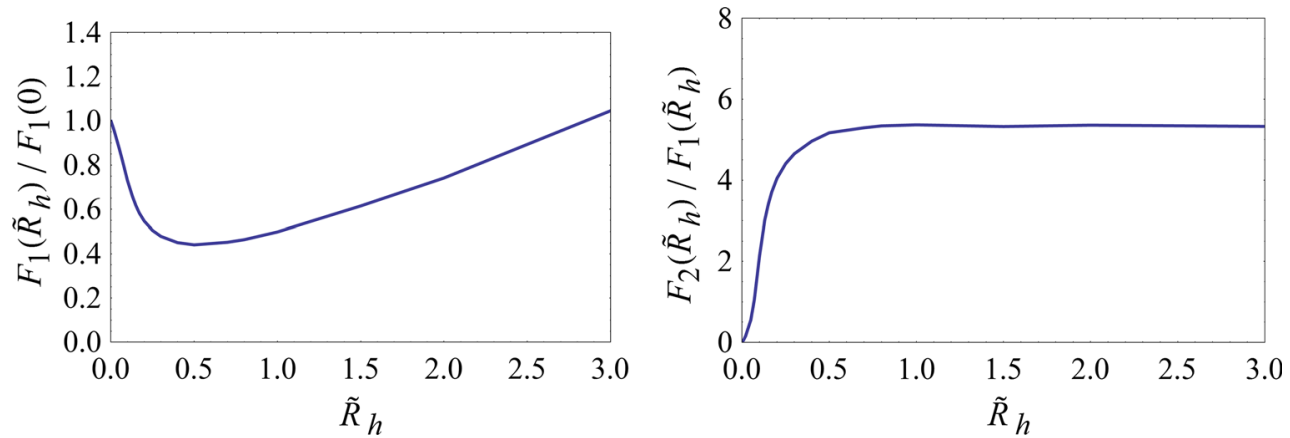

Fig. 3 The dimensionless quantities $F_{1}$ and $F_{2}$, that determine the ratio $\delta M_{\mathrm{tot}} / T$, as a function of the rescaled black-hole radius

and little progress can be achieved without assuming a specific mechanism for black-hole creation. In any case, putting everything together, we find that the logarithm of the probability of electroweak vacuum decay in the presence of black holes of typical radius $R_{\mathrm{h}}$ can be written as

$$
\begin{aligned}
\ln (N p P)= & 205+2 \ln \left(\frac{T}{M_{\mathrm{Pl}}}\right) \\
& +\ln p-\left(\frac{\delta M_{\mathrm{tot}}}{T}\right)_{0} A\left(R_{\mathrm{h}} T\right)\left[1+\xi B\left(R_{\mathrm{h}} T\right)\right],
\end{aligned}
$$

where $M_{\mathrm{Pl}}=(8 \pi G)^{-1 / 2} \simeq 2.43 \times 10^{18},\left(\delta M_{\mathrm{tot}} / T\right)_{0}$ is computed in the absence of black holes, and $A\left(R_{\mathrm{h}} T\right) \equiv$ $F_{1}\left(\tilde{R}_{\mathrm{h}}\right) / F_{1}(0), B\left(R_{\mathrm{h}} T\right) \equiv F_{2}\left(\tilde{R}_{\mathrm{h}}\right) / F_{1}\left(\tilde{R}_{\mathrm{h}}\right)$ are depicted in Fig. 3.

In Ref. [40] it was pointed out that $A\left(R_{\mathrm{h}} T\right)$ can lead to the reduction of the effect of $\left(\delta M_{\text {tot }} / T\right)_{0}$ by a factor of roughly 2 . Our present analysis demonstrates that the nonminimal coupling $\xi$ to gravity can have a more dramatic effect: For $\xi=-1 / B\left(R_{\mathrm{h}} T\right)$, the exponential suppression of the nucleation rate can be eliminated completely. Clearly, the saddle-point expansion around the bubble configuration breaks down before this point is reached. On the other hand, the presence of a large prefactor, coming from the huge number of causally independent regions in the early Universe, indicates that the electroweak vacuum is likely to become totally unstable.

As we have mentioned above, the largest uncertainty in the calculation is connected with the probability $p$ to find a black hole within each causally independent region at the time when the ambient temperature is $T$. A primordial black hole can form when the density fluctuations are sufficiently large for an overdense region to collapse [41-43]. It is usually assumed that its typical mass is of the order of its maximal possible mass. The latter is given by the total mass within the particle horizon $\sim M_{\mathrm{Pl}}^{2} / H$, while the maximal radius is $\sim 1 / H$. It is not clear, however, if these assumptions are consistent with the typical size of density fluctuations in the early
Universe, or the constraints imposed by the observations of the microwave background.

As a concrete application, we consider the scenario of Ref. [45], which is consistent with the current bounds on the size of primordial density fluctuations. It is assumed that the inflationary era is followed by a period during which the inflaton oscillates and decays into particles. The effective equation of state is similar to that of a matter-dominated Universe. It is possible, therefore, for perturbations that were generated during inflation to reenter the horizon, grow gravitationally and collapse into black holes. When the thermalization of the decay products takes place, the equation of state changes and the growth of perturbations is suppressed. In such a scenario, the black holes most relevant for our discussion are those produced just before thermalization, because they are the most massive ones. This must be contrasted with the scenario of the quantum decay of the electroweak vacuum in which the most relevant black holes are the light ones, because of their large Hawking temperature [37-39,45].

The modes that result in black-hole creation start from a primordial value $\delta \rho / \rho=\delta_{i} \sim 10^{-4}$ at horizon crossing and grow until $\delta \rho / \rho \sim 1$, at which point they decouple from the Hubble flow, turn around and collapse. The probability for them to form black holes has been estimated as $P_{\mathrm{BH}} \sim 2 \times 10^{-2}\left(R_{\mathrm{h}} / R\right)^{13 / 2}$ [49-51], with $R_{\mathrm{h}}$ the Schwarzschild and $R$ the turnaround radius. The black-hole mass is equal to that contained within the turnaround radius: $M=4 \pi \rho R^{3} / 3$. The Friedmann equation for a matterdominated era then gives: $R_{\mathrm{h}} / R \simeq H^{2} R^{2} \simeq \delta_{i}$. We can also deduce that $R_{\mathrm{h}} \simeq H^{2} R^{3} \simeq \delta_{i}^{3 / 2} / H$. As one expects $\sim(H R)^{-3}$ regions of size $R$ within a volume $\sim 1 / H^{3}$, the probability to find a black hole within the horizon is [45]

$p \sim 2 \times 10^{-22}\left(\frac{\delta_{i}}{10^{-4}}\right)^{5}$.

The bubble-nucleation rate is enhanced if, at the onset of the radiation-dominated era, there are black holes for which $\tilde{R}_{\mathrm{h}}=\kappa R_{\mathrm{h}} T \gtrsim 0.5$. At that time $\rho=\left(g_{*} \pi^{2} / 30\right) T^{4}$, where we take $g_{*}=106.75$, assuming only the particle content of the Standard Model. We obtain 
$\tilde{R}_{\mathrm{h}}=\kappa R_{\mathrm{h}} T \simeq \kappa\left(\frac{90}{g_{*} \pi^{2}}\right)^{1 / 2} \delta_{i}^{3 / 2} \frac{M_{\mathrm{Pl}}}{T} \simeq 0.1 \delta_{i}^{3 / 2} \frac{M_{\mathrm{Pl}}}{T}$.

If the reheating temperature is larger than $\sim 10^{-7} M_{\mathrm{Pl}}, \tilde{R}_{\mathrm{h}}$ is too small at the onset of the radiation-dominated era for the black holes to have a significant effect on the rate. At later times, black-hole creation is suppressed, the temperature drops, while the black-hole mass is reduced through evaporation. As a result $\tilde{R}_{\mathrm{h}}$ becomes even smaller.

It seems, therefore, that a high reheating temperature eliminates the danger posed by the presence of black holes. On the other hand, for $T \gtrsim 10^{-7} M_{\mathrm{Pl}}$, the typical mass of a black hole in this scenario is $M \lesssim 10^{27} \mathrm{GeV} \simeq 10^{3} \mathrm{~g}$. Such small black holes evaporate very quickly and are of little phenomenological interest. In this respect, the possibility of a reheating temperature smaller than $10^{-7} M_{\mathrm{Pl}}$ seems more exciting. As an example, let us consider the possibility of a reheating temperature $T \simeq 5 \times 10^{11} \mathrm{GeV}$, for which Eq. (26) with $\delta_{i} \simeq 10^{-4}$ gives $\tilde{R}_{\mathrm{h}} \simeq 0.5$, so that $A\left(R_{\mathrm{h}} T\right) \simeq 0.5$ and $B\left(R_{\mathrm{h}} T\right) \simeq 5.3$ in Eq. (24). Using Eq. (25), we obtain $\ln (N p P) \simeq 124-$ $0.5(1+5.3 \xi)\left(\delta M_{\text {tot }} / T\right)_{0}$, with $\left(\delta M_{\text {tot }} / T\right)_{0}$ computed in the absence of black holes. The probability becomes of order one for $\xi \lesssim-0.19+47 /\left(\delta M_{\mathrm{tot}} / T\right)_{0}$. The stability of the electroweak vacuum in the presence of primordial black holes imposes a strong constraint on the nonminimal coupling of the Higgs field to gravity, by forbidding this range.

Our discussion above neglects effects resulting from the accretion of the primordial plasma by the black hole. We expect that a stationary situation will develop with an accretion disc forming around the black hole. Even though the profile of the Higgs field will be distorted by this configuration, we still expect that the main effect will arise through the presence of the horizon and is captured by the boundary condition (10) that we assumed.

\section{Conclusions}

Given the absence of new physics in the LHC data, the stability of the SM electroweak vacuum has become a more pressing issue. Special conditions in the early Universe may not be left out when studying the rate of vacuum decay. As a particular example, black holes may trigger the decay, as first suggested in Refs. [37-39]. In this paper we have analyzed how the presence of primordial black holes may induce the SM electroweak vacuum decay in the early Universe at finite temperature. In particular, our findings indicate that a nonminimal, but renormalizable coupling between the SM Higgs field and gravity may alter considerably the decay rate.

Ultimately, the final fate of the electroweak vacuum is a model-dependent issue, which suffers from our ignorance of the precise early Universe dynamics and the exact black hole mass function as a function of time. Nevertheless, our results indicate that even moderate values of the coupling $\xi$ between the Higgs and gravity can render more stable or unstable the electroweak vacuum, depending on the sign of the coupling. While this calls for a refinement of our analysis, e.g. by understanding the role of the black-hole entropy on the problem, or by determining more precisely the probability $p$ for a black hole to exist in one of the many causally independent regions which are currently within our visible Universe, it demonstrates once more the importance of gravity for the issue of vacuum decay.

As we have mentioned before, we have not provided a formal treatment of the entropy of the Higgs configuration induced by the presence of the black hole. Instead, we have focused on the entropy related to the ambient temperature. This is not an unreasonable approximation in the scenario we discussed, because the leading effect is obtained at ambient temperatures $T / T_{\mathrm{H}}=\mathcal{O}\left(10^{2}\right)$. The black hole is not in equilibrium with the thermal environment, but we expect that it has a small effect on it. On the other hand, the black hole distorts the Higgs configuration through gravity, thus changing its energy, or free energy in a thermal environment.

A formal treatment of the problem faces several difficulties:

- In principle, one should start from the Euclidean action and impose a periodicity $\sim 1 / T$ in the time direction. On the other hand, the presence of the black hole requires a periodicity $\sim 1 / T_{\mathrm{H}}$, with $T_{\mathrm{H}}$ the Hawking temperature, in order to avoid a conical singularity on the background. The origin of the difficulty is the absence of thermal equilibrium, which necessitates an out-of-equilibrium analysis.

- The configuration we are interested in cannot be characterized as a black hole with hair. It is unstable by definition, as it must possess a negative mode. Therefore, it is not clear whether the notions of black-hole physics are applicable.

- Black-hole thermodynamics is well defined in the Einstein frame, while the natural setup for our problem is provided by the Jordan frame. Through a redefinition of the metric, it is possible to consider the problem in the Einstein frame. However, this would introduce unphysical couplings between the Higgs field and the matter sector.

The combination of all the above makes it hard to improve the robustness of our analysis. One could consider an alternative route through a statistical treatment, for multiple Higgs configurations that mimick the thermal bath. Even in such a case, one would have to define the statistical weight for each configuration through its energy or free energy, so that the problems discussed above would reappear in a different 
context. Despite these misgivings, the presence of an instability for a sufficiently negative nonminimal Higgs-gravity coupling remains a probable conclusion. Additional entropy contributions from the black-hole environment would further decrease the free energy $F=E-T S$ of the dominant configuration, making the transition to the true vacuum more likely.

Acknowledgements N.T. would like to thank A. Salvio, A. Strumia and A. Urbano for useful discussions. A.R. is supported by the Swiss National Science Foundation (SNSF), project Investigating the Nature of Dark Matter, Project number 200020-159223.

Open Access This article is distributed under the terms of the Creative Commons Attribution 4.0 International License (http://creativecomm ons.org/licenses/by/4.0/), which permits unrestricted use, distribution, and reproduction in any medium, provided you give appropriate credit to the original author(s) and the source, provide a link to the Creative Commons license, and indicate if changes were made. Funded by SCOAP ${ }^{3}$.

\section{References}

1. S.R. Coleman, F. De Luccia, Phys. Rev. D 21, 3305 (1980)

2. M. Sher, Phys. Rep. 179, 273 (1989)

3. P.B. Arnold, Phys. Rev. D 40, 613 (1989)

4. G. Altarelli, G. Isidori, Phys. Lett. B 337, 141 (1994)

5. J.A. Casas, J.R. Espinosa, M. Quiros, Phys. Lett. B 382, 374 (1996). arXiv:hep-ph/9603227

6. T. Hambye, K. Riesselmann, Phys. Rev. D 55, 7255 (1997). arXiv:hep-ph/9610272

7. G. Isidori, G. Ridolfi, A. Strumia, Nucl. Phys. B 609, 387 (2001). arXiv:hep-ph/0104016

8. J. Ellis, J.R. Espinosa, G.F. Giudice, A. Hoecker, A. Riotto, Phys. Lett. B 679, 369 (2009). arXiv:0906.0954 [hep-ph]

9. F. Bezrukov, M.Y. Kalmykov, B.A. Kniehl, M. Shaposhnikov, JHEP 1210, 140 (2012). arXiv:1205.2893 [hep-ph]

10. S. Alekhin, A. Djouadi, S. Moch, Phys. Lett. B 716, 214 (2012). arXiv:1207.0980 [hep-ph]

11. J. Elias-Miro, J.R. Espinosa, G.F. Giudice, G. Isidori, A. Riotto, A. Strumia, Phys. Lett. B 709, 222 (2012). arXiv:1112.3022 [hep-ph]

12. G. Degrassi, S. Di Vita, J. Elias-Miro, J.R. Espinosa, G.F. Giudice, G. Isidori, A. Strumia, JHEP 1208, 098 (2012). arXiv:1205.6497 [hep-ph]

13. D. Buttazzo, G. Degrassi, P.P. Giardino, G.F. Giudice, F. Sala, A. Salvio, A. Strumia, JHEP 1312, 089 (2013). arXiv:1307.3536 [hep$\mathrm{ph}]$

14. J.R. Espinosa, G.F. Giudice, A. Riotto, JCAP 0805, 002 (2008). arXiv:0710.2484 [hep-ph]

15. G. Isidori, V.S. Rychkov, A. Strumia, N. Tetradis, Phys. Rev. D 77, 025034 (2008). arXiv:0712.0242 [hep-ph]

16. L. Delle Rose, C. Marzo, A. Urbano, JHEP 1605, 050 (2016). arXiv:1507.06912 [hep-ph]

17. A. Salvio, A. Strumia, N. Tetradis, A. Urbano, JHEP 1609, 054 (2016). arXiv:1608.02555 [hep-ph]

18. A. Kobakhidze, A. Spencer-Smith, Phys. Lett. B 722, 130 (2013). arXiv:1301.2846 [hep-ph]
19. A. Kobakhidze, A. Spencer-Smith, Phys. Lett. B 722, 130 (2013). arXiv:1404.4709 [hep-ph]

20. K. Enqvist, T. Meriniemi, S. Nurmi, JCAP 1310, 057 (2013). arXiv:1306.4511 [hep-ph]

21. M. Fairbairn, R. Hogan, Phys. Rev. Lett. 112, 201801 (2014). arXiv:1403.6786 [hep-ph]

22. K. Enqvist, T. Meriniemi, S. Nurmi, JCAP 1407, 025 (2014). arXiv:1404.3699 [hep-ph]

23. K. Kamada, Phys. Lett. B 742, 126 (2015). arXiv:1409.5078 [hep$\mathrm{ph}$ ]

24. A. Hook, J. Kearney, B. Shakya, K.M. Zurek, JHEP 1501, 061 (2015). arXiv:1404.5953 [hep-ph]

25. M. Herranen, T. Markkanen, S. Nurmi, A. Rajantie, Phys. Rev. Lett. 113(21), 211102 (2014). arXiv:1407.3141 [hep-ph]

26. M. Herranen, T. Markkanen, S. Nurmi, A. Rajantie, Phys. Rev. Lett. 115, 241301 (2015). arXiv:1506.04065 [hep-ph]

27. A. Shkerin, S. Sibiryakov, Phys. Lett. B 746, 257 (2015). arXiv:1503.02586 [hep-ph]

28. J. Kearney, H. Yoo, K.M. Zurek, Phys. Rev. D 91(12), 123537 (2015). arXiv:1503.05193 [hep-th]

29. W.E. East, J. Kearney, B. Shakya, H. Yoo, K.M. Zurek, Phys. Rev. D 95(2), 023526 (2017)

30. W.E. East, J. Kearney, B. Shakya, H. Yoo, K.M. Zurek, Phys. Rev. D 95, 023526 (2017). arXiv:1607.00381 [hep-ph]

31. J.R. Espinosa, G.F. Giudice, E. Morgante, A. Riotto, L. Senatore, A. Strumia, N. Tetradis, JHEP 1509, 174 (2015). arXiv:1505.04825 [hep-ph]

32. A. Rajantie, S. Stopyra, Phys. Rev. D 95(2), 025008 (2017). arXiv: 1606.00849 [hep-th]

33. K. Enqvist, M. Karciauskas, O. Lebedev, S. Rusak, M. Zatta, JCAP 1611, 025 (2016). arXiv:1608.08848 [hep-ph]

34. Y. Ema, M. Karciauskas, O. Lebedev, M. Zatta, JCAP 1706(06), 054 (2017). arXiv:1703.04681 [hep-ph]

35. O. Czerwińska, Z. Lalak, M. Lewicki, P. Olszewski, JHEP 1610, 004 (2016). arXiv:1606.07808 [hep-ph]

36. B. Freivogel, G.T. Horowitz, S. Shenker, JHEP 0705, 090 (2007) arXiv:hep-th/0703146 [HEP-TH]

37. P. Burda, R. Gregory, I. Moss, Phys. Rev. Lett. 115, 071303 (2015). arXiv:1501.04937 [hep-th]

38. P. Burda, R. Gregory, I. Moss, JHEP 1508, 114 (2015). arXiv:1503.07331 [hep-th]

39. P. Burda, R. Gregory, I. Moss, JHEP 1606, 025 (2016). arXiv:1601.02152 [hep-th]

40. N. Tetradis, JCAP 1609(09), 036 (2016). arXiv:1606.04018 [hep$\mathrm{ph}]$

41. Y.B. Zeldovich, I.D. Novikov, Sov. Astron. A J. 10, 602 (1967)

42. S. Hawking, Mon. Not. R. Astron. Soc. 152, 75 (1971)

43. B.J. Carr, S.W. Hawking, Mon. Not. R. Astron. Soc. 168, 399 (1974)

44. R. Bousso, S.W. Hawking, Phys. Rev. D 54, 6312 (1996). arXiv:gr-qc/9606052

45. D. Gorbunov, D. Levkov, A. Panin, JCAP 1710(10), 016 (2017). arXiv:1704.05399 [astro-ph.CO]

46. G.W. Gibbons, S.W. Hawking, Phys. Rev. D 15, 2738 (1977)

47. J.R. Espinosa, M. Garny, T. Konstandin, A. Riotto, Phys. Rev. D 95(5), 056004 (2017). arXiv:1608.06765 [hep-ph]

48. P.B. Arnold, S. Vokos, Phys. Rev. D 44, 3620 (1991)

49. M.Y. Khlopov, A.G. Polnarev, Phys. Lett. 97B, 383 (1980)

50. A.G. Polnarev, M.Y. Khlopov, Sov. Phys. Usp. 28, 213 (1985)

51. A.G. Polnarev, M.Y. Khlopov, Usp. Fiz. Nauk 145, 369 (1985) 